\title{
Ventricular septal defect causing tricuspid regurgitation with consequent pulmonary hypertension and right ventricular hypertrophy
}

\author{
Mahmoud Abdelnabi MD, Abdallah Almaghraby MD
}

CASE

A 25-year-old man complaining of grade II dyspnea was referred for transthoracic echocardiography (TTE) for further assessment of his tricuspid valve before surgical repair/replacement. He was previously diagnosed with isolated severe tricuspid regurgitation causing pulmonary hypertension and was scheduled for tricuspid repair/replacement, if needed. On clinical examination, he had a harsh systolic murmur at the lower left sternal border and apex. A TTE revealed thickened tricuspid valve leaflets with severe tricuspid regurgitation with consequent pulmonary hypertension with right ventricular hypertrophy. A small perimembranous ventricular septal defect (VSD) partially closed by an aneurysmally dilated septal leaflet of the tricuspid valve was detected resulting in loss of coaptation of the tricuspid valve leaflets. (Figure 1 panel A-D, Video 1) The tricuspid regurgitation with consequent pulmonary hypertension was induced by two different mechanisms: first, by the high-velocity flow of the peri-membranous VSD distorting the tricuspid valve;

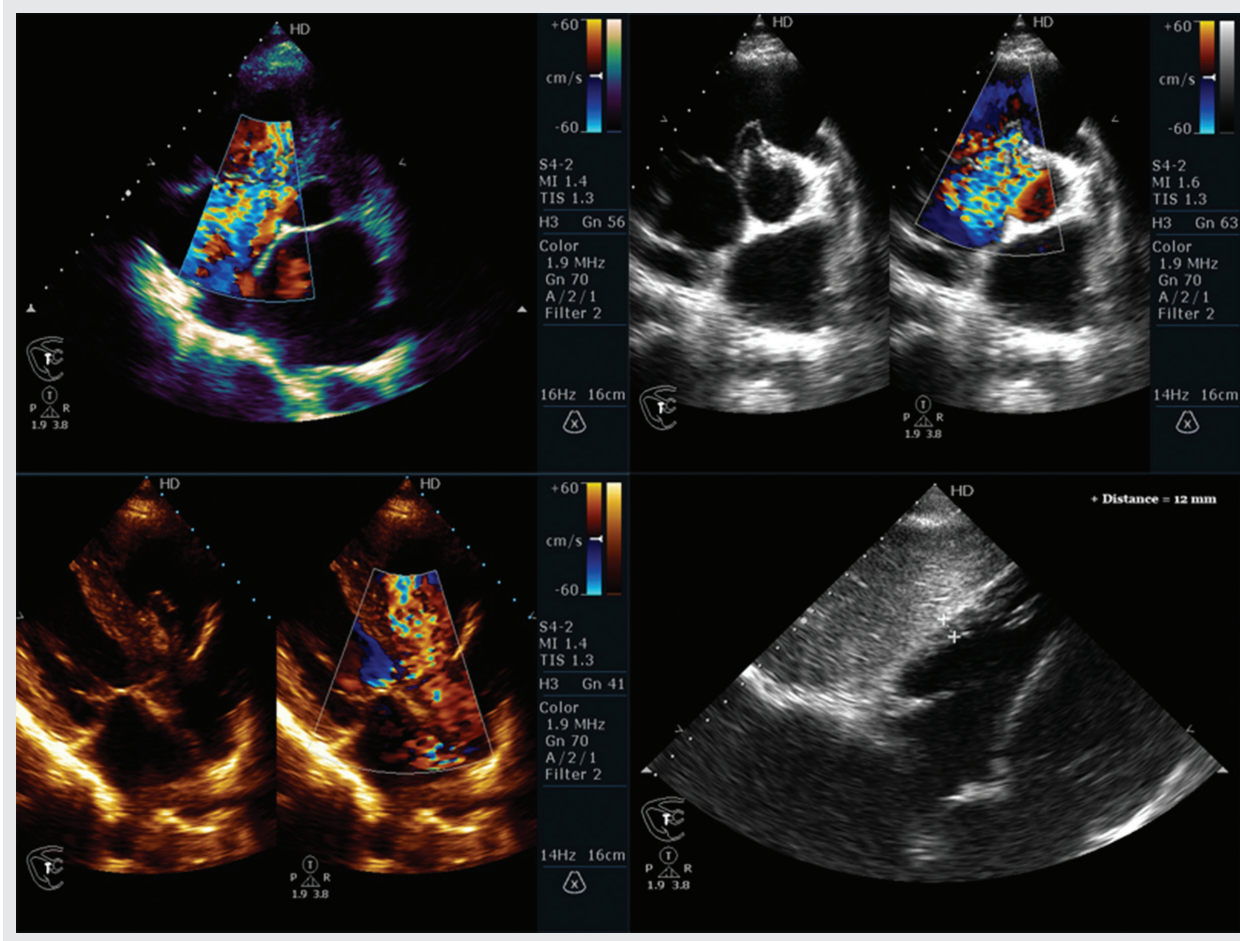

Figure 1. Panel A-D: Transthoracic echocardiography showing small perimembranous ventricular septal defect causing severe tricuspid regurgitation, pulmonary hypertension, and right ventricular hypertrophy.

Video 1. Transthoracic echocardiography of peri-membranous ventricular septal defect causing tricuspid regurgitation and pulmonary hypertension.

Corresponding author: Mahmoud Hassan Abdelnabi Contact Information: Mahmoud.hassan.abdelnabi@ outlook.com

DOI: $10.12746 /$ swrccc.v9i40.869 
second, by the Venturi effect that induces suction of the septal leaflet to partially close the defect resulting in loss of coaptation. The clinical decision was changed from tricuspid valve repair/replacement to VSD percutaneous closure. Follow-up echocardiography after VSD closure revealed mild residual tricuspid regurgitation and resolution of pulmonary hypertension.

\section{Discussion}

Several mechanisms have been proposed to explain tricuspid regurgitation associated with perimembranous VSD. First, it can be induced by high velocity from the VSD resulting in distortion of the anterior leaflet of the tricuspid valve redirecting the flow towards the right atrium; second, septal leaflet aneurysm which is induced by the Venturi effect of the shunt and partially closes the shunt but results in the loss of complete closure of tricuspid valve during systole with subsequent tricuspid regurgitation. ${ }^{1}$ Perimembranous VSD closure either by surgery or device occlusion is usually associated with a total resolution of tricuspid regurgitation. ${ }^{2}$

Consent: Informed written consent was obtained from the patient.

Keywords: Echocardiography, peri-membranous ventricular septal defect, tricuspid regurgitation, pulmonary hypertension
Article citation: Abdelnabi M, Almaghraby A. Ventricular septal defect causing tricuspid regurgitation with consequent pulmonary hypertension and right ventricular hypertrophy. The Southwest Respiratory and Critical Care Chronicles 2021;9(40):77-78

From: Cardiology and Angiology Unit (MA), Clinical and Experimental Internal Medicine Department, Medical Research Institute, Alexandria University, Alexandria, Egypt; Department of Internal Medicine (MA), Texas Tech University Health Sciences Center, Lubbock, Texas; Department of Cardiology (AA), Faculty of Medicine, Alexandria University, Alexandria, Egypt

Submitted: 4/18/2021

Accepted: 5/16/2021

Reviewer: Aliakbar Arvandi MD

Conflicts of interest: none

This work is licensed under a Creative Commons Attribution-ShareAlike 4.0 International License.

\section{REFERENCES}

1. Hagler DJ, Squarcia U, Cabalka AK, et al. Mechanism of tricuspid regurgitation in paramembranous ventricular septal defect. J American Soc Echocardiography 2002;15(4):364-368.

2. Kumar V, Aggarwal N, Swamy A, et al. Disappearing high velocity severe tricuspid regurgitation following Ventricular septal defect device closure. Indian Heart J 2017;69(3): 402-404. 